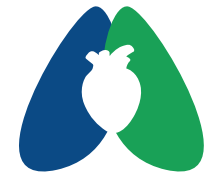

ASSOBRAFIR C I Ê N C I A

\section{Correlação entre mobilidade toracoabdominal, força muscular respiratória, pico de fluxo de tosse e as complicações pulmonares no pós- operatório de cirurgias abdominais}

\author{
Correlation between toracoabdominal mobility, respiratory muscle \\ strength, peak cough flow and pulmonary complications post- \\ surgery abdominal
}

\begin{abstract}
Ingrid Stefany Bittencourt ${ }^{1}$ (D); Matheus Rocha Martin ${ }^{1}$ (D); Priscila Santos Soares ${ }^{1}$ (D); Marilucia Reis dos Santos² (D); Júlio David Nascimento da Rocha² (D); Jorge Luis Motta dos Anjos ${ }^{3}$; Fabiane Costa Santos Fontoura' (1); Caroline Ferreira Guerreiro' ${ }^{1}$ (D)

'Departamento de Fisioterapia, Faculdade de Tecnologia e Ciências (FTC), Salvador, BA, Brasil 2Programa de residência em Fisioterapia em Terapia Intensiva e Emergência, Hospital Geral Roberto Santos (HGRS), Salvador, BA, Brasil

${ }^{3}$ Comissão de Residências Multiprofissionais em Saúde (COREMU), Hospital Geral Roberto Santos (HGRS), Salvador, BA, Brasil.
\end{abstract}

\begin{abstract}
Resumo
Introdução: Cirurgias abdominais comumente comprometem a biomecânica respiratória, o que pode resultar em diversas complicações pulmonares pós-operatórias. Objetivo: Verificar a correlação entre mobilidade toracoabdominal, força muscular respiratória, pico de fluxo de tosse e as complicações pulmonares no pós-operatório de cirurgias abdominais. Métodos: Trata-se de um estudo observacional, transversal, com amostra por conveniência de pacientes submetidos a cirurgias abdominais, adultos, de ambos os sexos, internados em unidades de terapia intensiva (UTI) de um hospital da rede pública estadual em Salvador-BA. Foram utilizados a cirtometria (axilar, xifoide e umbilical), a manovacuometria e o pico de fluxo de tosse (PFT) para avaliar a função respiratória. Para análise da correlação entre as três variáveis foi utilizado o coeficiente de Spearman. Resultados: A amostra foi composta por 19 pacientes, sendo $13(68,4 \%)$ do sexo masculino com média de idade de $53,89 \pm 20,91$ anos. Verificou-se redução da mobilidade toracoabdominal $(<4 \mathrm{~cm})$, da força muscular respiratória (PImáx 44,47 $\pm 20,13 \mathrm{cmH}_{2} \mathrm{O}$, PEmáx 48,16 $\pm 25,34 \mathrm{cmH}_{2} \mathrm{O}$ ) e do PFT $(137,1 \pm 87,1 \mathrm{~L} / \mathrm{min})$ nos indivíduos estudados, contudo não houve diferença entre os grupos com e sem complicações pulmonares, assim como não houve correlação entre as variáveis respiratórias e as complicações pulmonares pós-operatórias. Conclusão: Indivíduos submetidos a cirurgias abdominais, mesmo as de baixa incisão, apresentam redução da mobilidade toracoabdominal, força muscular respiratória e capacidade de tossir, contudo não há correlação entre as variáveis respiratórias e as complicações pulmonares no momento pós-operatório.
\end{abstract}

Descritores: Teste de Função Pulmonar; Complicações Pós Operatórias; Força Muscular.

Como citar: Bittencourt IS; Martin MR; Soares PS; Santos MR; Rocha JDN; Anjos et al. Correlação entre mobilidade toracoabdominal, força muscular respiratória, pico de fluxo de tosse e as complicações pulmonares no pósoperatório de cirurgias abdominais. ASSOBRAFIR Ciênc. 2020;11:e42085. https:// doi.org/10.47066/2177-9333.AC.2020.0015

Submissão em: Novembro 12, 2020 Aceito em: Abril 09, 2021

Estudo realizado em: Hospital Geral Roberto Santos, Salvador, Bahia, Brasil. Aprovação ética: CAAE

69898717.0.0000.5028 do Hospital Geral Roberto Santos, $n^{\circ} 066144 / 2017$.

*Autor correspondente:

Caroline Ferreira Guerreiro. E-mail:

carol.guerreiro@yahoo.com.br

\begin{abstract}
Background: Abdominal surgeries commonly compromise respiratory biomechanics, which can result in several postoperative pulmonary complications. Aim: To verify the correlation between thoracoabdominal mobility, respiratory muscle strength, peak cough flow and pulmonary complications in the postoperative period of abdominal surgery. Methods: This is an observational, cross-sectional study with a convenience sample of patients undergoing abdominal surgeries, adults, of both sexes, admitted to intensive care units (ICU) of a state public network hospital in Salvador-BA. Cirtometry (axillary, xiphoid and umbilical), manovacuometry and peak cough flow (PFT) were used to assess respiratory function. Spearman's coefficient was used to analyze the correlation between the three variables. Results: The sample consisted of 19 patients, 13 (68.4\%) males with a mean age of $53.89 \pm 20.91$ years. There was a reduction in thoracoabdominal mobility $(<4 \mathrm{~cm})$, respiratory muscle strength (MIP $44.47 \pm 20.13 \mathrm{cmH} 2 \mathrm{O}$, MEP $48.16 \pm 25.34 \mathrm{cmH} 2 \mathrm{O}$ ) and PFT $(137.1 \pm 87.1 \mathrm{~L} / \mathrm{min})$ in the individuals studied, however, there was no difference between the groups with and without pulmonary complications, as well as no
\end{abstract} sob a mesma licença. 
correlation between respiratory variables and postoperative pulmonary complications. Conclusion: Individuals submitted to abdominal surgeries, even those with low incisions, have reduced thoracoabdominal mobility, respiratory muscle strength and coughing capacity, but there is no correlation between respiratory variables and pulmonary complications in the postoperative period.

Keywords: Lung Function Test; Postoperative Complications; Muscle Strength.

\section{INTRODUÇÃO}

Cirurgias abdominais são realizadas rotineiramente para o tratamento e diagnóstico de muitas doenças. No entanto, apesar da necessidade de abordagem, seja de urgência ou eletiva, envolve alterações da biomecânica respiratória, que resultam na redução da força muscular, dos volumes e capacidades pulmonares, comprometendo a eficiência da tosse ${ }^{1-3}$. Alterações que consequentemente favorecem o surgimento de complicações pulmonares pósoperatórias (CPPO), resultando em pior desfecho clinico durante o internamento ${ }^{4-6}$.

As CPPO são doenças ou condições inesperadas que ocorrem até trinta dias após uma cirurgia, tais como: a pneumonia, derrame pleural e o tempo prolongado em ventilação mecânica invasiva (VMI) ${ }^{7-9}$. Adicionalmente, existem os fatores contribuintes para etiologia das CPPO, como as variáveis cirúrgicas, comorbidades prévias e uso de bloqueadores neuromusculares, que associados implicam no aumento da mortalidade perioperatória, do tempo de internamento e das taxas de re-hospitalização ${ }^{10-12}$.

Embora seja reconhecida sua relevância clínica, os fatores que causam as complicações respiratórias ainda não estão plenamente definidos, inviabilizando a definição de medidas com foco na intervenção precoce durante o período de internamento. Diante disso, o objetivo deste estudo foi verificar a correlação entre mobilidade toracoabdominal, força muscular respiratória, pico de fluxo de tosse e as complicações pulmonares no pósoperatório de cirurgias abdominais

\section{MÉTODOS}

Trata-se de um estudo observacional, transversal, com amostra por conveniência de pacientes submetidos a cirurgias abdominais, adultos, de ambos os sexos, internados em unidades de terapia intensiva (UTI) de um hospital da rede pública estadual em Salvador-BA. O projeto foi aprovado pelo Comitê de Ética e Pesquisa do Hospital Geral Roberto Santos (HGRS) sob parecer 066144/2017 e CAAE 69898717.0.0000.5028. A pesquisa foi delineada de acordo com as resoluções do CNS N. 466/12 e N. 510/16 conforme os princípios éticos de autonomia, beneficência, não maleficência e justiça. Todos os indivíduos aceitaram participar assinando o termo de consentimento livre e esclarecido (TCLE).

Foram excluídos da pesquisa indivíduos mantidos em VMI por tempo maior que 48 horas após admissão na UTI, que apresentaram alteração da função cognitiva, dificultando a aplicação das técnicas e dos instrumentos de avaliação propostos, doenças neurológicas associadas, história de traumatismo crânio-encefálico (TCE), cirurgia torácica prévia e qualquer alteração motora que impedisse a realização dos exames, assim como restrições médicas.

Para a coleta de dados, foi construída uma ficha onde foram registrados os seguintes dados: nome, idade, sexo, cor e comorbidades auto relatadas. Além de dados secundários obtidos em prontuários e exames complementares como: queixa principal, data de admissão no hospital, diagnóstico clínico, perfil (clínico ou cirúrgico), necessidade de intubação, tempo de VMI, data de entrada e saída da UTI, desfecho na saída (alta, óbito, transferência para outra instituição), bem como as CPPO: pneumonia, infecção traqueobrônquica, atelectasia, broncoespasmo, disfunção diafragmática, embolia pulmonar, derrame pleural, insuficiência respiratória aguda, pneumotórax e fístulas broncopleurais.

As variáveis primárias avaliadas foram a mobilidade toracoabdominal, força muscular respiratória e capacidade de tossir avaliados nas primeiras 48 horas após a cirurgia, sendo considerados os seguintes parâmetros: pressão inspiratória máxima (PImáx), pressão expiratória máxima (PEmáx) e pico de fluxo de tosse (PFT), que foram avaliados através de parâmetros da manovacuometria e peak flow, respectivamente, sendo a mobilidade toracoabdominal mensurada através da cirtometria.

A cirtometria foi mensurada de acordo com as descrições de Kakizaki e colaboradores ${ }^{13}$ utilizando-se uma fita métrica sobre a caixa torácica do voluntário em três regiões diferentes: axilar, xifoide e umbilical. Cada medida foi obtida solicitando ao paciente que expirasse o máximo possível e em seguida fizesse uma inspiração máxima seguida de outra expiração máxima. Essas medidas foram repetidas duas vezes seguidas sendo considerado a média dos dois valores obtidos. A mobilidade torácica foi considerada reduzida quando a variação foi inferior a 4cm em pelo menos uma região do tórax ou abdômen ${ }^{14-16}$.

A manovacuometria foi realizada utilizando um manovacuômetro analógico -150/ +150 cm/ $\mathrm{H}_{2} \mathrm{O}$ (VENTCARE INDUSTRIA E COMERCIO LTDA, Brasil) para avaliar as pressões inspiratórias e expiratórias máximas, segundo técnica descrita por Black and Hyatt (1969). Das variáveis referentes às pressões respiratórias máximas foi considerado o valor previsto para a idade e sexo segundo Costa e colaboradores ${ }^{17}$ e o maior valor obtido das três manobras de pressão inspiratória máxima $\left(\mathrm{cmH}_{2} \mathrm{O}\right.$ negativo), bem como as de pressão expiratória máxima ( $\mathrm{cmH}_{2} \mathrm{O}$ positivo). 
A PImáx foi aferida solicitando ao paciente que expirasse próximo ao volume residual (VR) e puxasse o ar rápida e profundamente. A PEmáx foi aferida solicitando ao mesmo que puxasse o ar próximo a capacidade pulmonar total (CPT), em seguida, soltasse o ar o mais forte e rápido possível. O paciente foi posicionado com a cabeceira elevada no mínimo em $60^{\circ}$, com a cabeça em posição neutra e apoiada no leito, sendo utilizado um clipe nasal para evitar escape de ar. Foram feitas três manobras, com variação entre elas dentro de $10 \%$, sendo escolhido o maior valor obtido ${ }^{18}$.

Foi utilizado o aparelho "peak flow" para mensurar o PFT, sendo solicitado ao participante que puxasse o ar até próximo a CPT e realizasse uma manobra de tosse pelo bocal com o máximo de força e rapidez possível, não podendo colocar a língua na frente do bocal. 0 procedimento foi repetido por três vezes, utilizando-se o maior valor obtido para a análise estatística. Os valores de PFT abaixo de 160L/min têm sido associados à ineficiência da tosse em realizar a remoção de secreções devido a uma possível deterioração da musculatura respiratória durante períodos de infecção respiratória, e valores iguais ou acima de $270 \mathrm{~L} / \mathrm{min}$ são utilizados para identificar pacientes que possuem uma tosse eficaz ${ }^{19}$.

A avaliação nutricional foi realizada através do índice de massa corpórea (IMC), calculado como a razão entre o peso pelo quadrado da altura $\left(\mathrm{kg} / \mathrm{m}^{2}\right)$, coletados em prontuário. Esta medida é utilizada para diagnóstico nutricional, que estabelece um IMC inferior a $18,5 \mathrm{~kg} / \mathrm{m}^{2}$ para baixo peso, entre 18,5 e $24,9 \mathrm{~kg} / \mathrm{m}^{2}$ para eutrofia, sobrepeso quando o valor do IMC estiver entre 24,9 e $29,9 \mathrm{~kg} / \mathrm{m}^{2}$, e obesidade acima de $30 \mathrm{~kg} / \mathrm{m}^{2}$. (OMS, 2015). Este dado foi utilizado no presente estudo para caracterização da amostra e controle do viés de confundimento, visto que o componente abdominal pode influenciar a avaliação da função respiratória.

Os exames respiratórios foram realizados por três avaliadores treinados, sob orientação e responsabilidade de um profissional com formação e experiência em fisioterapia respiratória. Não foram observados sinais de fadiga durante os exames e nenhum dos participantes solicitou interrupção dos testes e desistência da pesquisa.

Para análise dos dados utilizou-se de variáveis de natureza qualitativa e quantitativa. Algumas variáveis tiveram diferentes categorização e natureza a depender das análises empregadas. Dentre as variáveis qualitativas destaca-se: sexo (masculino e feminino); faixa etária (18 a 24 anos; 25 a 59 anos e 60 a 76 anos) ou (18 a 59 anos e 60 a 76 anos); raça (branco, preto, parto) ou (branco e preto/pardo); IMC (eutrófico, sobrepeso e obesidade); hábito tabagista (sim e não); hábito etilista (sim e não); HAS (sim e não); cardiopatia (sim e não); DM (sim e não); doença renal (sim e não); AVE (sim e não); neoplasia (sim e não); tipo de cirurgia (cirurgia abdominal alta e cirurgia abdominal baixa) e CPPO (com ou sem complicações). Além disso, foram calculadas médias e desvio-padrão de variáveis quantitativas como idade, IMC, cirtometria toracoabdominal, a força muscular respiratória e o PFT e analisado a frequência relativa do uso de sedativo, antibióticos, corticóides e DVA.

Para caracterizar a população em estudo foram calculadas frequências absolutas e relativas das características sociodemográficas, hábitos de vida e variáveis que caracterizam a saúde ou processos patológicos dos pacientes.

Para compreensão da relação entre as CPPO e variáveis demográficas, hábitos de vida e história pregressa da doença dos pacientes, utilizou-se de variáveis binárias para análise através do teste de qui-quadrado de Pearson considerando um $\mathrm{p}$-valor de $5 \%$ de significância.

Para observar a relação entre a cirtometria toracoabdominal, a força muscular respiratória, PFT e as CPPO foi utilizado o teste de Mann-Whitney considerando um p-valor de $5 \%$ de significância e a correlação de Spearman com um nível de significância de 5\%.

Com base na literatura, considerou-se coeficientes entre 0,00 a 0,25 indicando pouca ou nenhuma correlação; aqueles entre 0,25 a 0,50 sugerindo um grau fraco de correlação; valores entre 0,50 e 0,75 indicando uma correlação de moderada a boa; e valores acima de 0,75 foram considerados de boa a excelente correlação ${ }^{20}$.

Todas as análises foram executadas através do software Stata 12 e as tabelas foram construídas no Excel for Windows.

\section{RESULTADOS}

No presente estudo, 29 indivíduos foram admitidos na UTI no pós-operatório de cirurgias abdominais, sendo 10 excluídos, destes, 2 por alteração da função cognitiva, 2 por doenças neurológicas associadas, 1 devido à instabilidade hemodinâmica, 2 por permanecerem em VMI prolongada, 1 por alteração motora que impediu a realização dos exames e 2 devido ao tempo de internação para avaliação (acima de 48h), totalizando uma amostra de 19 indivíduos avaliados.

Houve predominância do sexo masculino $(68,4 \%)$, com média de idade de 53,89 $\pm 20,91$ anos, prevalência da raça parda (47,4\%), com média de IMC $24,78 \pm 4,04 \mathrm{Kg} / \mathrm{m}^{2}$, caracterizando a amostra com o perfil eutrófico. Além disso, constatou-se que a maioria dos participantes não eram tabagistas $(68,4 \%)$ e etilistas $(73,7 \%)$, sendo observado que $73,7 \%$ não apresentavam HAS e $89,5 \%$ não relataram cardiopatia ou DM. Não houve relato sobre doenças renais e acidente vascular encefálico (AVE). Verificou-se que $89,5 \%$ não possuía neoplasias e houve um predomínio de cirurgias abdominais baixa $(57,9 \%)$. A média do tempo de internamento na UTI foi de 3,58 $\pm 2,81$ dias e, entre as CPPO desenvolvidas, constatou-se que a pneumonia correspondeu a 87,5\% (n=7) e o pneumotórax a 12,5\% ( $n=1)$ (Tabela 1). 
Tabela 1. Características sociodemográficas e clinicas da amostra $(\mathrm{N}=19)$.

\begin{tabular}{|c|c|c|}
\hline Variáveis & $\mathrm{n}(\%)$ & Média \pm DP \\
\hline \multicolumn{3}{|l|}{ SEXO } \\
\hline Masculino & $13(68,4 \%)$ & \\
\hline Feminino & $6(31,6 \%)$ & \\
\hline FAIXA ETÁRIA (ANOS) & & $53,89 \pm 20,91$ \\
\hline 18 a 24 & $4(21,1 \%)$ & \\
\hline 25 a 59 & $4(21,1 \%)$ & \\
\hline 60 a 76 & $11(57,8 \%)$ & \\
\hline \multicolumn{3}{|l|}{ RAÇA } \\
\hline Branco & $6(31,6 \%)$ & \\
\hline Preto & $4(21,0 \%)$ & \\
\hline Pardo & $9(47,4 \%)$ & \\
\hline IMC & & $24,78 \pm 4,04$ \\
\hline Eutrófico & $10(52,6 \%)$ & \\
\hline Sobrepeso & $8(42,1 \%)$ & \\
\hline Obesidade & $1(5,3 \%)$ & \\
\hline \multicolumn{3}{|l|}{ TIPO DE CIRURGIA } \\
\hline Cirurgia abdominal alta & $8(42,1 \%)$ & \\
\hline Cirurgia abdominal baixa & $11(57,9 \%)$ & \\
\hline \multicolumn{3}{|l|}{ HÁBITO TABAGISTA } \\
\hline Sim & $6(31,6 \%)$ & \\
\hline Não & $13(68,4 \%)$ & \\
\hline \multicolumn{3}{|l|}{ HÁBITO ETILISTA } \\
\hline Sim & $5(26,3 \%)$ & \\
\hline Não & $14(73,7 \%)$ & \\
\hline \multicolumn{3}{|l|}{ CPPO } \\
\hline Pneumonia & $7(87,5 \%)$ & \\
\hline Pneumotórax & $1(12,5 \%)$ & \\
\hline
\end{tabular}

Legenda: DP = desvio padrão; IMC = índice de massa corpórea.

Conforme apresentado na Figura 1, entre os pacientes avaliados, 63,2\% fizeram uso de antibióticos, 5,3\% usaram corticoide e 15,8\% necessitaram de drogas vasoativas. Durante o procedimento cirúrgico $47,4 \%$ dos pacientes fizeram uso de sedação e $42 \%$ dos pacientes permaneceram na VMI, com média de 27士16,97 horas.

A Tabela 2 apresenta os dados dos indivíduos referentes a cirtometria toracoabdominal, força muscular respiratória e PFT. Em relação a cirtometria, as variações axilar, xifoide e umbilical corresponderam a valores abaixo de $4 \mathrm{~cm}$, caracterizando uma mobilidade toracoabdominal reduzida. Em relação à média da força muscular respiratória, as pressões respiratórias apresentaram-se

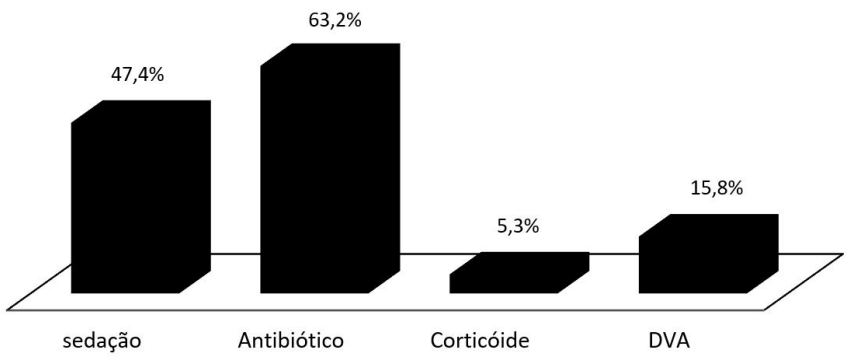

Figura 1. Frequência do uso de medicamentos em pacientes submetidos a cirurgias abdominais ( $\mathrm{N}=19)$.

Tabela 2. Dados descritivos da cirtometria toracoabdominal, força muscular respiratória e pico de fluxo de tosse dos indivíduos submetidos a cirurgias abdominais ( $N=19)$.

\begin{tabular}{lc}
\hline \multicolumn{1}{c}{ Variáveis } & Média $( \pm \mathrm{DP})$ \\
\hline Cirtometria toracoabdominal & \\
Variação axilar $(\mathrm{cm})$ & $3,06( \pm 1,97)$ \\
Variação xifoide $(\mathrm{cm})$ & $2,03( \pm 0,9)$ \\
Variação umbilical $(\mathrm{cm})$ & $0,97( \pm 0,81)$ \\
Força muscular respiratória & \\
PImáx $\left(\mathrm{cmH}_{2} \mathrm{O}\right)$ & $44,47( \pm 20,13)$ \\
Medida & $127,27( \pm 56,89)$ \\
Previsto & 35 \\
\% previsto & \\
PEmáx $\left(\mathrm{cmH}{ }_{2} \mathrm{O}\right)$ & $48,16( \pm 25,34)$ \\
Medida & $103,87( \pm 26,23)$ \\
Previsto & 46 \\
\% previsto & \\
Fluxos pulmonares & $137,1( \pm 87,1)$ \\
PFT (L/min) &
\end{tabular}

Legenda: PImáx - pressão inspiratória máxima; PEmáx - pressão expiratória máxima; PFT - pico de fluxo de tosse. Costa et al. 2010.

reduzidas. A PImáx foi equivalente a $35 \%$ do valor previsto $\left(44,47 \pm 20,13 \mathrm{cmH}_{2}\right.$ O) e a PEmáx equivalente a $46 \%$ do valor previsto $\left(48,16 \pm 25,34 \mathrm{cmH}_{2} \mathrm{O}\right)$. A média do PFT encontrada foi de $137,1( \pm 87,1) \mathrm{L} / \mathrm{min}$ caracterizando a tosse como ineficaz.

Quando comparados os pacientes que apresentaram ou não CPPO não houve diferença entre os grupos em relação as variáveis sexo, idade, raça, hábito tabagista e etilista e o tipo de cirurgia realizada (Tabela 3).

Não houveram diferenças entre as medidas de cirtometria toracoabdominal, força muscular respiratória e o PFT entre os indivíduos que apresentaram ou não CPPO durante o período de internamento na UTI (Tabela 4).

A Tabela 5 evidencia que não houve correlação entre as medidas de cirtometria toracoabdominal, força muscular 
Correlação entre mobilidade toracoabdominal, força muscular respiratória, pico de fluxo de tosse e as complicações pulmonares no pós-operatório de cirurgias abdominais

Tabela 3. Distribuição das variáveis demográficas e hábitos de vida de acordo com a presença de complicações pulmonares no pósoperatório de cirurgias abdominais ( $\mathrm{N}=19)$.

\begin{tabular}{|c|c|c|c|}
\hline \multicolumn{4}{|c|}{ CPPO } \\
\hline & $\operatorname{Sim}(n=8)$ & Não $(n=11)$ & p-valor \\
\hline SEXO & & & 0,60 \\
\hline Masculino & $75,0 \%$ & $63,6 \%$ & \\
\hline Feminino & $25,0 \%$ & $36,4 \%$ & \\
\hline FAIXA ETÁRIA & & & 0,12 \\
\hline 18 a 59 anos & $62,5 \%$ & $27,3 \%$ & \\
\hline 60 a 76 anos & $37,5 \%$ & $72,7 \%$ & \\
\hline RAÇA & & & 0,14 \\
\hline Branco & $50,0 \%$ & $18,2 \%$ & \\
\hline Preto/Pardo & $50,0 \%$ & $81,8 \%$ & \\
\hline HÁBITO TABAGISTA & & & 0,60 \\
\hline Sim & $25,0 \%$ & $36,4 \%$ & \\
\hline Não & $75,0 \%$ & $63,6 \%$ & \\
\hline HÁBITO ETILISTA & & & 0,91 \\
\hline Sim & $25,0 \%$ & $27,3 \%$ & \\
\hline Não & $75,0 \%$ & $72,7 \%$ & \\
\hline TIPO DE CIRURGIA & & & 0,73 \\
\hline Cirurgia abdominal alta & $37,5 \%$ & $45,4 \%$ & \\
\hline Cirurgia abdominal baixa & $62,5 \%$ & $54,6 \%$ & \\
\hline \multicolumn{4}{|l|}{ DOR } \\
\hline Ausência de dor & $37,5 \%$ & $45,4 \%$ & \\
\hline Presença de dor & $62,5 \%$ & $36,4 \%$ & \\
\hline
\end{tabular}

Legenda: Teste Qui-Quadrado de Pearson. CPPO = complicações pulmonares pós-operatórias. $p<0,05$.

Tabela 4. Média da cirtometria toracoabdominal, força muscular respiratória e pico de fluxo de tosse de acordo com a presença de complicações pulmonares no pós-operatório de cirurgias abdominais ( $\mathrm{N}=19)$.

\begin{tabular}{|c|c|c|c|}
\hline \multicolumn{4}{|c|}{ CPPO } \\
\hline & $\operatorname{Sim}(n=8)$ & Não (n=11) & \multirow{2}{*}{ p-valor } \\
\hline & Média \pm DP & Média \pm DP & \\
\hline \multicolumn{4}{|c|}{ CIRTOMETRIA TORACOABDOMINAL } \\
\hline Variação axilar & $3,2 \pm 1,77$ & $3,0 \pm 2,25$ & 0,62 \\
\hline Variação xifoide & $1,8 \pm 0,51$ & $2,2 \pm 1,06$ & 0,62 \\
\hline Variação umbilical & $1,2 \pm 0,89$ & $0,8 \pm 0,65$ & 0,48 \\
\hline \multicolumn{4}{|c|}{ FORÇA MUSCULAR RESPIRATÓRIA } \\
\hline PImáx $\left(\mathrm{cm} / \mathrm{H}_{2} \mathrm{O}\right)$ & $43,7 \pm 14,08$ & $45,0 \pm 24,29$ & 0,90 \\
\hline PEmáx $\left(\mathrm{cm} / \mathrm{H}_{2} \mathrm{O}\right)$ & $56,2 \pm 17,88$ & $42,3 \pm 29,01$ & 0,17 \\
\hline \multicolumn{4}{|c|}{ FLUXOS PULMONARES } \\
\hline $\mathrm{PFT}$ (L/min) & $156,2 \pm 122,23$ & $123,2 \pm 51,88$ & 0,53 \\
\hline
\end{tabular}

Legenda: Teste Qui-Quadrado de Pearson. CPPO = complicações pulmonares pós-operatórias. $p<0,05$ 
Tabela 5. Correlação entre cirtometria toracoabdominal, força muscular respiratória, pico de fluxo de tosse e as complicações pulmonares no pós-operatório de cirurgias abdominais ( $\mathrm{N}=19)$.

\begin{tabular}{lcc}
\hline \multicolumn{3}{c}{ CPPO } \\
\hline Variação axilar $(\mathrm{cm})$ & $-0,06$ & p-valor \\
Variação xifóide $(\mathrm{cm})$ & 0,10 & 0,82 \\
Variação umbilical $(\mathrm{cm})$ & $-0,10$ & 0,69 \\
PImáx $\left(\mathrm{cm} / \mathrm{H}_{2} \mathrm{O}\right)$ & $-0,07$ & 0,68 \\
PEmáx $\left(\mathrm{cm} / \mathrm{H}_{2} \mathrm{O}\right)$ & $-0,37$ & 0,77 \\
PFT $(\mathrm{L} / \mathrm{min})$ & $-0,22$ & 0,12 \\
\hline
\end{tabular}

Legenda: $r=$ coeficiente de Spearman. $\mathrm{CPPO}=$ complicações pulmonares pós-operatórias; PImáx= pressão inspiratória máxima; $P$ Emáx= pressão expiratória máxima; PFT= pico de fluxo de tosse. p<0,05.

respiratória, pico de fluxo de tosse e as CPPO de cirurgias abdominais.

\section{DISCUSSÃO}

Os resultados do presente estudo não evidenciam correlação entre mobilidade toracoabdominal, força muscular respiratória, pico de fluxo de tosse e as complicações pulmonares no pós-operatório de cirurgias abdominais. Este estudo é pioneiro no que diz respeito a correlação entre as medidas de mobilidade toracoabdominal, força muscular respiratória, pico de fluxo de tosse e as CPPO de cirurgias abdominais, não sendo encontrado até o momento outros resultados comparativos.

A abordagem abdominal envolve a manipulação de vísceras e músculos, inclusive respiratórios, como o diafragma, sendo esperado que o paciente apresente respiração mais superficial e diminuição de volumes pulmonares, o que não foi o evidenciado em nossos achados. Isso pode ser explicado pela predominância do tipo de incisão cirúrgica baixa, que comparado às cirurgias abdominais altas, apresentam menor impacto na função respiratória ${ }^{21}$.Rocatto e colaboradores por meio de um estudo prospectivo experimental, avaliaram 38 pacientes no pré e pós-operatório de cirurgia abdominal alta e constataram que houveram reduções significativas das medidas de cirtometria axilar, mamilar e xifoide entre os dois momentos, com aumento significativo após o protocolo fisioterapêutico ${ }^{22}$.

Estudos demostram que existem repercussões pulmonares no pós-operatório imediato de cirurgias abdominais altas, o que pode ser evidenciado pela diminuição da expansibilidade torácica e da força muscular respiratória23-25. Fato que pode ser explicado pela proximidade entre o local da abordagem cirúrgica e pela localização de músculos envolvidos com a respiração. Contudo, no presente estudo não foram encontradas diferenças entre as medidas de cirtometria toracoabdominal entre os grupos de pacientes que apresentaram ou não CPPO de cirurgias abdominais. Assim, pode-se inferir que possivelmente houveram reduções da mobilidade toracoabdominal nesses indivíduos, o que remete a necessidade de atenção e estratégias preventivas para evitar complicações futuras.

Embora as pressões respiratórias máximas estivessem reduzidas no momento pós-operatório, os resultados do presente estudo não mostram diferença entre os grupos com e sem complicações pulmonares, divergindo do estudo de Veloso e colaboradores que demonstraram que o enfraquecimento dos músculos respiratórios após a cirurgia abdominal pode levar a complicações pós-operatórias, dificultando o trabalho respiratório e aumentando o tempo de internação hospitalar ${ }^{26}$. Santos e colaboradores também divergem dos achados do presente estudo, na medida em que evidenciaram que pacientes submetidos a colecistectomia apresentam redução significativa da PImáx e PEmáx, sendo esta um importante fator de risco para o aparecimento de complicações respiratórias no pós-operatório ${ }^{27}$.

Em relação a avaliação da tosse, observou-se neste estudo a predominância da tosse ineficaz nos doentes, sem diferença quanto a presença de complicações pulmonares. Colucci e colaboradores ${ }^{28}$, por meio de um estudo prospectivo de coorte, constataram que a eficácia da tosse estava prejudicada após cirurgia abdominal superior, uma vez que o PFT sofreu redução de até $72 \%$ em relação às medições observadas antes da cirurgia. A dor pós-operatória é um fator importante que pode influenciar o ato de tossir e levar a complicações pulmonares após as cirurgias $^{16}$. Entretanto, dos participantes deste estudo, a maioria (58\%) não referia dor no momento da avaliação, inferindo-se desta forma, uma relação da fraqueza muscular respiratória com o comprometimento da tosse.

Oliveira e colaboradores constataram que na cirurgia abdominal alta a incidência de complicações pulmonares varia de $10 \%$ a $81 \%$, enquanto que o risco dessas complicações na cirurgia abdominal inferior varia de $0 \%$ para $5 \%{ }^{29}$, divergindo dos resultados do presente estudo, no qual $62,5 \%$ dos pacientes que apresentaram algum tipo de CPPO foram submetidos a cirurgia abdominal baixa. Esses dados sugerem que mesmo em indivíduos submetidos a cirurgia abdominal baixa, a avaliação e o acompanhamento da função respiratória durante o período de internamento, torna-se de suma importância na redução das CPPO.

Haines e colaboradores avaliaram 72 pacientes submetidos a cirurgias abdominais de grande porte e verificaram que 39\% apresentaram complicações pulmonares. A diferença média do tempo de internação entre os que apresentaram ou não algum tipo daquela complicação foi de 5,9 dias $^{30}$. No presente estudo, observou-se que não houveram diferenças no tempo de internamento na UTI entre os grupos que desenvolveram ou não CPPO, fato que pode ser justificado pela possibilidade do tratamento das pneumonias à nível 
domiciliar, não impactando no tempo de permanência na UTI.

Silva e colaboradores destacaram que as CPPO ocorrem após $25 \%$ a $50 \%$ dos procedimentos cirúrgicos maiores ${ }^{31}$, corroborando com os achados deste estudo, onde aproximadamente $42 \%$ dos pacientes avaliados apresentaram algum tipo de complicação pulmonar. Fatores que ressaltam a necessidade do desenvolvimento de estratégias preventivas ainda no período de internação hospitalar, mesmo em casos de cirurgias com incisão baixa, a fim de minimizar as alterações respiratórias e evitar ou reduzir as possíveis complicações pulmonares, as taxas de morbimortalidade e os custos hospitalares.

O presente estudo apresenta algumas limitações, tais como o pequeno tamanho amostral, que pode ser justificado pelo curto período para coleta de dados e a não realização das avaliações respiratórias pré-operatórias, devido a impossibilidade de acesso ao setor específico, limitando desta forma uma análise estatística mais detalhada.

\section{CONCLUSÃO}

O presente estudo verificou que não houve correlação entre as variáveis respiratórias e as complicações pulmonares no pós-operatório de cirurgias abdominais. Contudo, os achados sugerem que indivíduos submetidos a cirurgias abdominais, mesmo as de baixa incisão, podem apresentar redução da mobilidade toracoabdominal, da força muscular respiratória e da capacidade de tossir, sendo necessário, a realização de mais estudos com este fim.

\section{FONTE DE FINANCIAMENTO}

Nada a declarar.

\section{CONFLITO DE INTERESSES}

Nada a declarar.

\section{AGRADECIMENTOS}

Agradecemos ao HGRS, principalmente a equipe multiprofissional da UTI cirúrgica, pelo acolhimento e presteza durante o período da pesquisa em campo. A todos os participantes, essenciais ao desenvolvimento da pesquisa, a eterna nossa gratidão visto que mesmo em um momento de fragilidade de saúde consideraram a contribuição ao ensino, aprendizado e a ciência em prol da comunidade.

\section{REFERÊNCIAS}

1. Kumar AS, Alaparthi GK, Augustine AJ, Pazhyaottayil ZC, Ramakrishna A, Krishnakumar SK. Comparison of flow and volume incentive spirometry on pulmonary function and exercise tolerance in open abdominal surgery: A randomized clinical trial. J Clin Diagn Res. 2016;10(1):KC0106. PMid:26894090.

2. Ávila AC, Fenili R. Incidência e fatores de complicações pulmonares pós-operatórias em pacientes submetidos à cirurgias de tórax e abdomen. Rev. Col. Bras. 2017;44(3):28492. http://dx.doi.org/10.1590/0100-69912017003011.

3. Langeron O, Carreira S, le Saché F, Raux M. Postoperative pulmonary complications updating. Ann Fr Anesth Reanim. 2014;33(7-8):480-3. http://dx.doi.org/10.1016/j. annfar.2014.07.741. PMid:25168300.

4. Zraier S, Haouache H, Dhonneur G. Which preoperative respiratory evaluation? Ann Fr Anesth Reanim. 2014;33(78):453-6. http://dx.doi.org/10.1016/j.annfar.2014.07.743. PMid:25168302.

5. Yang CK, Teng A, Lee DY, Rose K. Pulmonary complications after major abdominal surgery: National Surgical Quality Improvement Program analysis.J Surg Res. 2015;198(2):441-9. http://dx.doi.org/10.1016/j.jss.2015.03.028. PMid:25930169.

6. Boden I, Browning L, Skinner EH, Reeve J, El-Ansary $D$, Robertson IK, et al. The LIPPSMACK POP (Lung Infection Prevention Post Surgery - Major Abdominal with Pre-Operative Physiotherapy) trial: study protocol for a multi-centre randomised controlled trial. Trials. 2015;16(1):573-88. http://dx.doi.org/10.1186/s13063-0151090-6. PMid:26666321.

7. Bastos LC, Pereira PC, Moraes FC, Oliveira LHS. Pressões inspiratoria e expiratoria maximas no pré e pós-operatório de cirugias abdominais altas. Rev Univ Vale Rio Verde. 2018;16(1):1. http://dx.doi.org/10.5892/ruvrd.v16i1.3639.

8. Karcz M, Papadakos PJ. Respiratory complications in the postanesthesia care unit: A review of pathophysiological mechanisms. Can J Respir Ther. 2013;49(4):21-9. PMid:26078599.

9. Miskovic A, Lumb AB. Postoperative pulmonar complications. Br J Anaesth. 2017;118(3):317-34. http://dx.doi.org/10.1093/ bja/aex002. PMid:28186222.

10. Sanguineti VA, Wild JR, Fain MJ. Management of postoperative complications: general approach. Clin Geriatr Med. 2014;30(2):261-70. http://dx.doi.org/10.1016/j. cger.2014.01.005. PMid:24721365.

11. Fernandes SCS, Santos RS, Giovanetti EA, Taniguchi C, Silva CSM, Eid RAC, et al. Impact of respiratory therapy in vital capacity and functionality of patients undergoing abdominal surgery. Einstein (Sao Paulo). 2016;14(2):202-7. http://dx.doi. org/10.1590/S1679-45082016AO3398. PMid:27462894.

12. Taylor A, DeBoard Z, Gauvin JM. Prevention of postoperative pulmonary complications. Surg Clin North Am. 2015;95(2):237-54. http://dx.doi.org/10.1016/j. suc.2014.11.002. PMid:25814104.

13. Kakizak IF, Shibuya M, Yamazaki T, Yamada M, Suzuki H, Homma L. Preliminary report on the effects of respiratory muscle stretch gymnastics on chest wall mobility in patients with chronic obstructive pulmonary disease. Respir Care. 1999;44:409-14.

14. Tasca C, Schuster RC, Alvarenga LFC. Força muscular respiratória e mobilidade torácica em portadores de doença de Parkinson. Rev. enferm. Atenção Saúde. 2014;12(42):510.

15. Basso RP, Regueiro EMG, Jamami M, Di Lorenzo VAP, Costa D. Relação da medida da amplitude tóraco-abdominal de adolescentes asmáticos e saudáveis com seu desempenho físico. Fisioter Mov. 2011;24(1):107-14. http://dx.doi. org/10.1590/S0103-51502011000100012. 
16. Ramos ML, Neves DR, Lima VP, Orsini M, Machado D, Bastos VHV, et al. Análise de parâmetros pneumofuncionais em pacientes com doença de Parkinson: estudo piloto. Rev Bras Neurol. 2014;50(2):38-43.

17. Costa D, Gonçalves HA, Lima LP, Ike D, Cancelliero KM, Montebelo MIL. Novos valores de referência para pressões respiratórias máximas na população brasileira. J Bras Pneumol. 2010;36(3):306-12. http://dx.doi.org/10.1590/ S1806-37132010000300007. PMid:20625667.

18. Souza FSP, Silva BG, Echevarria LB, Silva AA, Pessoti E, Forti EMP. Fisioterapia respiratória associada à pressão positiva nas vias aéreas na evolução pós-operatória da cirurgia bariátrica. Fisioter Pesqui. 2012;19(3):204-9. http://dx.doi. org/10.1590/S1809-29502012000300003.

19. Freitas FS, Parreira VF, Ibiapina CC. Aplicação clínica do pico de fluxo da tosse: uma revisão de literatura. Fisioter Mov. 2010;23(3):495-502. http://dx.doi.org/10.1590/S010351502010000300016.

20. Portney LG, Watkins MP. Foundations of Clinical Research Applications to Practice. 1997; Connecticut, USA: Appleton \& Lange.

21. Scheeren CFC, Goncalves JJS. Avaliação comparativa da função ventilatória através do pico de fluxo expiratório no pré e pós-operatório imediatos de pacientes submetidos a procedimentos cirúrgicos eletivos de andar superior de abdome. Rev Col Bras Cir. 2016;43(3):165-70. http://dx.doi. org/10.1590/0100-69912016003007. PMid:27556540.

22. Rocatto GEGD, Oliveira HJC, Segundo AS, Da Silva AMC, Neto EM, Nasrala MLS. Fisioterapia respiratória no pós-operatório imediato de colecistectomia convencional. Fisioterapia Brasil. 2014;15(3):195-9.

23. Rê A, Ono LM, Gram ST, Saltiel RV, Shivinski CIS, Matte $D L$, et al. Repercussões da colecistectomia aberta na mobilidade diafragmática e toracoabdominal. RBM. 2012;69(10):261-6.

24. Trevisan ME, Soares JC, Rondinel TZ. Efeitos de duas técnicas de incentivo respiratório na mobilidade tóraco abdominal após cirurgia abdominal alta. Fisioter Pesqui. 2010;17(4):3226. http://dx.doi.org/10.1590/S1809-29502010000400007.

25. Caldeira VS, Starling CC, Britto RR, Martins JA, Sampaio RF, Parreira VF. Reliability and accuracy of cirtometry in healthy adults. J Bras Pneumol. 2007;33(5):519-26. http://dx.doi. org/10.1590/S1806-37132007000500006. PMid:18026649.

26. Veloso APLR, Cusmanich KC. Avaliação da mobilidade toracoabdominal dos obesos no pré-operatório de cirurgia bariátrica. ABCD Arq. Bras. Cir. Dig. 2016;29(1):3942. http://dx.doi.org/10.1590/0102-6720201600s10011. PMid:27683774.

27. Santos CA, Rabelo BAS, Borges DL, Silva TM. Avaliação da força muscular respiratória de pacientes submetidos à colecistectomia videolaparoscópica. ASSOBRAFIR Ciência. 2016;7(1):35-42.

28. Colucci DB, Fiore JF Jr, Paisani DM, Risso TT, Colucci M, Chiavegato LD, et al. Cough Impairment and Risk of Postoperative Pulmonary Complications After Open Upper Abdominal Surgery. Respir Care. 2015;60(5):673-8. http:// dx.doi.org/10.4187/respcare.03600. PMid:25669217.

29. Oliveira MA, Vidotto MC, Nascimento AO, Almeida R, Santoro IL, Sperandio EF, et al. Avaliação dos volumes pulmonares, capacidade vital e força dos músculos respiratórios no pós-operatório de cirurgia na coluna cervical, torácica e lombar. Sao Paulo Med J. 2015;133(5):388-93. http://dx.doi. org/10.1590/1516-3180.2014.00252601. PMid:26648426.

30. Haines KJ, Skinner EH, Berney S, and the Austin Health POST Study Investigators. Association of postoperative pulmonary complications with delayed mobilisation following major abdominal surgery: an observational cohort study. Physiotherapy. 2013;99(2):119-25. http://dx.doi. org/10.1016/j.physio.2012.05.013. PMid:23219632.

31. Silva DR, Gazzana MB, Knorst MM. Valor dos achados clínicos e da avaliação funcional pulmonar pré-operatórios como preditores das complicações pulmonares pós-operatórias. Rev Assoc Med Bras. 2010;56(5):551-7. http://dx.doi. org/10.1590/S0104-42302010000500016. PMid:21152827. 